\title{
Brain Structure and Function in Patients after Metal-on-Metal Hip Resurfacing
}

\author{
M.J. Clark, J.R. Prentice, N. Hoggard, M.N. Paley, M. Hadjivassiliou, and J.M. Wilkinson
}

\begin{abstract}
BACKGROUND AND PURPOSE: Hip prostheses that use a metal-on-metal articulation expose the brain to elevated metal concentrations that, in acute excess due to prosthesis malfunction, is associated with neurologic damage, including visual and hearing loss and motor deficits. Here, we examined whether chronic exposure to lower elevated metal levels, typical of well-functioning prostheses, also affects brain structure and function.

MATERIALS AND METHODS: We compared brain volumes, metal deposition, and gray matter attenuation by MR imaging and clinical neurologic function in patients 8 years after receiving a metal-on-metal hip resurfacing versus a matched group of patients with the same duration exposure to a conventional hip prosthesis.

RESULTS: Twenty-nine patients ( 25 men; mean, age $59 \pm 7$ years) after metal-on-metal hip resurfacing and 29 patients (25 men; $59 \pm 8$ years) after total hip arthroplasty were compared. Whole blood cobalt and chromium concentrations were 5-10 times higher in the metal-on-metal hip resurfacing group $(P<.0001)$. Occipital cortex gray matter attenuation tended to be lower $(P<.005$ uncorrected, $P>$ .05 corrected), and the optic chiasm area tended to be lower (mean difference, $-2.7 \mathrm{~mm}^{2} ; P=.076$ ) in the metal-on-metal hip resurfacing group. Subgroup analyses in 34 patients (17 per group), after exclusion of primary ocular pathology, showed the same trend in gray matter attenuation in the occipital cortex and basal ganglia and a smaller optic chiasm in the metal-on-metal hip resurfacing group (mean difference, $-3.9 \mathrm{~mm}^{2} ; P=.048$ ). No other structural or functional differences were found between the groups.
\end{abstract}

CONCLUSIONS: Chronic exposure to metal-on-metal hip resurfacing is associated with subtle structural change in the visual pathways and the basal ganglia in asymptomatic patients.

ABBREVIATIONS: $\mathrm{Co}=$ cobalt; $\mathrm{Cr}$ = chromium; MOMHR = metal-on-metal hip resurfacing; PRIME = partially refocused interleaved multiple-echo sequences; THA = total hip arthroplasty; UPDRS = Unified Parkinson's Disease Rating Scale

C obalt (Co) and Chromium (Cr) are common component materials of joint prostheses, and these metals are released by many designs of joint replacement but particularly from those with a metal-on-metal articulating bearing. ${ }^{1,2} \mathrm{Co}$ and $\mathrm{Cr}$ cross the blood-brain barrier and may be deposited in the brain. ${ }^{3,4} \mathrm{Co}$ and $\mathrm{Cr}$ are toxic to neuronal cells in rats, causing brain cell apoptosis

Received November 14, 2013; accepted after revision February 13, 2014.

From the Departments of Human Metabolism (M.J.C., J.R.P., J.M.W.) and Cardiovascular Science (N.H., M.N.P.), University of Sheffield, The Medical School, Sheffield, United Kingdom; Department of Neurology (M.H.), Sheffield Teaching Hospitals National Health Service Foundation Trust, Royal Hallamshire Hospital, Sheffield, United Kingdom; and Department of Orthopaedics (J.M.W.), Sheffield Teaching Hospitals National Health Service Foundation Trust, Northern General Hospital, Sheffield, United Kingdom.

This study was funded by Sheffield Musculoskeletal Biomedical Research Unit through its core grant from the National Institute of Health Research in the United Kingdom.

The National Institute of Health Research in the United Kingdom had no role in the design and conduct of the study; collection, management, analysis, and interpretation of the data; or preparation, review, or approval of the manuscript. mediated by the $\alpha$ subunit of hypoxia inducible factor- 1 pathway. ${ }^{5-7}$ Metal toxicity presenting with neurologic deficit is reported in association with malfunctioning hip prostheses and excessive circulating Co and $\mathrm{Cr}^{8}{ }^{8}$ These neurologic deficits include visual and hearing loss, peripheral sensory and motor defects, tremor, and cognitive decline. ${ }^{9,10}$

In the United States, $>500,000$ patients have received hip replacements by using a metal-on-metal bearing, and most have well-functioning devices. ${ }^{11}$ Well-functioning prostheses also release metal species into the systemic circulation, albeit at much lower concentrations than those associated with malfunctioning prostheses. Steady-state median blood Co and Cr concentrations during 10 years in patients with well-functioning devices are typ-

Please address correspondence to J. Mark Wilkinson, MD, Department of Human Metabolism, University of Sheffield, DU24, The Medical School, Beech Hill Rd Sheffield, S10 2RX, United Kingdom; e-mail: j.m.wilkinson@sheffield.ac.uk

- Indicates open access to non-subscribers at www.ajnr.org

http://dx.doi.org/10.3174/ajnr.A3922 
ically between 1.5 and $2.5 \mu \mathrm{g} / \mathrm{L}$, and are 5 to 10 -fold higher than normal physiologic concentrations. ${ }^{12,13}$ The effect on the brain of prolonged exposure to these moderately elevated concentrations of circulating metal for many years is unclear.

We examined whether prolonged exposure to circulating metal after metal-on-metal hip resurfacing (MoMHR) is associated with differences in brain structure and function assessed by MR imaging and clinical neurologic assessment in a group of clinically healthy patients after MoMHR versus an age-, sex-, diagnosis-, and time since surgery-matched group of patients who received a conventional total hip arthroplasty (THA) that did not use a metal-on-metal bearing to determine whether this choice of prosthesis has implications for brain health. We, therefore, chose demographically similar patients with conventional THA for the control group, rather than use background population controls, to minimize potential confounding due to brain structural change associated with underlying diagnosis or surgical treatment per se. ${ }^{14}$

\section{MATERIALS AND METHODS \\ Subjects}

We undertook this single-center cross-sectional study at a teaching hospital in the United Kingdom. Patients were recruited as part of a study examining the systemic effects of metal exposure after MoMHR ( $n=35$ patients in each group). ${ }^{15}$ The study was approved by a National Research Ethics Service Research Ethics Committee, and all patients provided written informed consent before participation. All patients underwent clinical neurologic examination, including an assessment of extrapyramidal tract function by using the Unified Parkinson's Disease Rating Scale (UPDRS), Part 2 (activities of daily living) and Part 3 (motor). The UPDRS is a standardized scale used to assess the severity of Parkinson disease symptoms and has previously been applied to the quantitation of movement disorders associated with metal neurotoxicity. ${ }^{16,17}$ Blood and plasma Co and Cr levels were measured by inductively coupled plasma-mass spectroscopy, as described previously. ${ }^{15}$

\section{MR Imaging Acquisition}

MR images were obtained by using a 3T system (Intera; Phillips Healthcare, Best, Netherlands) with an 8-channel sensitivity encoding head coil. The imaging protocol comprised structural, functional, and partially refocused interleaved multiple-echo (PRIME) sequences. 3D T1 magnetization-prepared rapid acquisition of gradient echo images were acquired for volumetric analysis $\left(\mathrm{TR}=10.5 \mathrm{~ms}, \mathrm{TE}=4.8 \mathrm{~ms}\right.$, flip angle $=8^{\circ}, 187$ sagittal sections with $0.8-\mathrm{mm}$ thickness, $256 \times 205 \mathrm{~mm}$ FOV, acquired voxel size $=0.8 \times 0.8 \times 0.8 \mathrm{~mm}$, scan time $=5$ minutes 30 seconds). MR images were aligned parallel to the internal auditory meatus in the coronal plane and parallel with the midline of the brain in the sagittal plane. Sagittal images were reconstructed to continuous coronal images with a $0.9-\mathrm{mm}$ section thickness.

The PRIME acquisition sequence was used to simultaneously measure quantitative relaxation rates, $\mathrm{R} 2$ and $\mathrm{R} 2^{*}$, and hence $\mathrm{R} 2{ }^{\prime}$, which is related to magnetic inhomogeneity caused by metal deposition. The sequence comprised 5 spin-echoes with 9 gradient recalled-echoes per spin-echo, leading to a total of 45 images per section (relaxation time $=3000 \mathrm{~ms}$; spin-echo times $=30,60,90,120$, and $150 \mathrm{~ms}$; gradient-echo times at 3-ms increments for 9 echoes spaced symmetrically around the spin-echo; recon matrix $=512 \times$ 512 ; flip angle $=90^{\circ}$; acquired voxel size $=1.44 \times 1.8 \times 2.5 \mathrm{~mm} ; 14$ coronal sections with a section thickness of $2.5 \mathrm{~mm}$; scanning time, 5 minutes 0 seconds). All analyses were made by using the fMRI of the Brain (FMRIB) Software Library (FSL, Version 4.1.1; http://www. fmrib.ox.ac.uk/fsl), apart from metal deposition relaxation time measurements made using the PRIME sequence and optic chiasm area made using in-house software.

\section{Data Analysis}

Brain volume and subcortical nuclear volumes were measured by using the FSL FMRIB Integrated Registration and Segmentation Tool (FIRST) and automated segmentation and bias field correction tool (FAST) after deletion of nonbrain tissue from images by using the Brain Extraction Tool. ${ }^{18}$ FAST was applied to estimate total brain gray and white matter, and FIRST was used to measure brain stem volumes and the volumes of 7 subcortical regions, including the amygdala, caudate nucleus, putamen, thalamus, pallidum, hippocampus, and nucleus accumbens. FSLstats (http://www2. warwick.ac.uk/fac/sci/statistics/staff/academic-research/nichols/ scripts/fsl/fslstats.sh) was used to output numeric data. All measured volumes were corrected for intracranial volume.

Metal deposition in the brain was assessed by using a relativequantitative method with in-house Matlab software (Version 7.9; MathWorks, Natick, Massachusetts). We have previously used this method to measure brain iron concentration. ${ }^{19-21}$ Here we applied it to $\mathrm{Co}$ and $\mathrm{Cr}$ quantitation because both $\mathrm{Co}$ and $\mathrm{Cr}$ in their stable states (Co II and Cr III) are paramagnetic. ${ }^{22}$ Briefly, the method involves processing the images by using a nonlinear least-squares fitting routine to calculate values for R2'. The PRIME sequence provides a complete set of simultaneously acquired and registered $\mathrm{T} 2$ and $\mathrm{T} 2{ }^{*}$ contrast-weighted images for each section, allowing calculation of the $\mathrm{T} 2$ and $\mathrm{T} 2{ }^{*}$ relaxation times. T2 was calculated from the peak signal of the 5 acquired spin-echoes, and $\mathrm{T} 2{ }^{*}$ was calculated from the peak signal from the 5 echoes, including the first spin-echo and subsequent 4 gradient echoes. R2' was calculated from the formula $\mathrm{R} 2^{\prime}=\left(1 / \mathrm{T} 2^{\star}-1 / \mathrm{T} 2\right)$ by using the difference in the fits to the spin-echo and gradient-echo exponential decay curves from selected regions of interest. This measure indicates the additional inhomogeneity generated by the metal susceptibility difference from normal tissue and minimizes effects due to changes in the $\mathrm{T} 2$ relaxation time from other potential sources.

Quantitative measures of gray matter attenuation were made by using voxel-based morphometry (FSL-VBM, Version 1.1; http://fsl.fmrib.ox.ac.uk/fsl/fsl-4.1.9/fslvbm/index.html). ${ }^{23,24}$ Structural images had the brain extracted by using the Brain Extraction Tool. ${ }^{25}$ The gray matter was then segmented and registered to the Montreal Neurological Institute 152 standard space by using nonlinear registration. ${ }^{26}$ The resulting images were averaged to create a study-specific template to which the native gray matter images were nonlinearly registered and corrected for local expansion or contraction. The modulated gray matter images were then smoothed with an isotropic Gaussian kernel with a $\sigma$ of $3 \mathrm{~mm}$. Differences in gray matter attenuation were calculated by threshold free-cluster enhancement by using permutation-based nonparametric testing (5000 permutations) by using the Ran- 
Table 1: Patient characteristics ${ }^{a}$

\begin{tabular}{lccc}
\hline \multicolumn{1}{c}{ Characteristic } & MoMHR $(\boldsymbol{n}=\mathbf{2 9})$ & THA $(\boldsymbol{n}=\mathbf{2 9})$ & $\boldsymbol{P}$ Value \\
\hline Age at surgery (yr) & $58.9 \pm 6.8$ & $59.2 \pm 7.5$ & .87 \\
Sex (M/F) & $25: 4$ & $25: 4$ & 1.00 \\
Time since surgery (yr) & $8.0 \pm 1.7$ & $7.7 \pm 2.5$ & .51 \\
UPDRS: Part 2 (ADLs) deficit & $0(0-1)$ & $1(0-2)$ & .34 \\
UPDRS: Part 3 (motor) deficit & $0(0-1)$ & $0(0-1)$ & .76 \\
Whole-blood cobalt $(\mu \mathrm{g} / \mathrm{L})$ & $1.72(1.10-6.01)$ & $0.38(0.33-0.50)$ & $<.0001$ \\
Whole-blood chromium $(\mu \mathrm{g} / \mathrm{L})$ & $1.42(1.01-3.86)$ & $<0.30(<0.30$ to $<0.30)$ & $<.0001$ \\
Plasma cobalt $(\mu \mathrm{g} / \mathrm{L})$ & $1.66(0.89-4.47)$ & $<0.30(<0.30$ to $<0.30)$ & $<.0001$ \\
Plasma chromium $(\mu \mathrm{g} / \mathrm{L})$ & $2.52(1.60-.13)$ & $<0.30(<0.30$ to $<0.30)$ & $<.0001$ \\
\hline
\end{tabular}

Note:-ADL indicates activities of daily living.

${ }^{\text {a }}$ Data are mean \pm SD or median (interquartile range). Analyses of continuous data are MoMHR versus THA by unpaired $t$ test or the Mann-Whitney $U$ test; analysis of sex distribution is by the Fisher exact test.

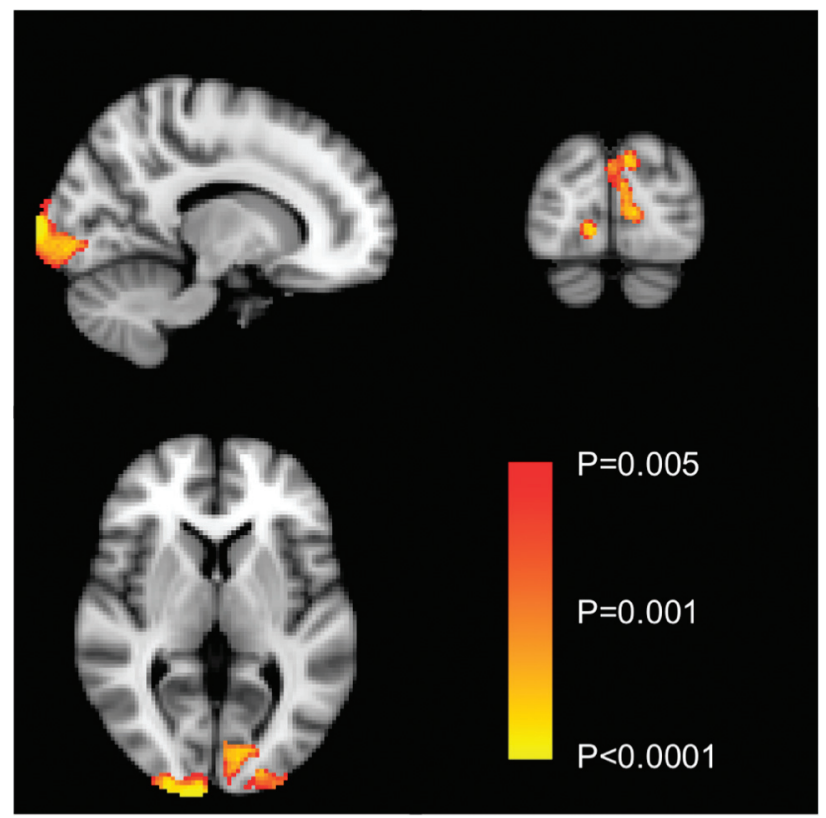

FIG 1. Voxel-based morphometric analysis of differences in brain gray matter attenuation between the MoMHR and THA groups before exclusion of patients with primary ocular pathology. Comparative areas of gray matter loss in the MoMHR group are highlighted by using the color bar across the significance value range $P<.05$ to $P<.001$ (uncorrected).

domize tool within FSL (http://fsl.fmrib.ox.ac.uk/fsl/fslwiki/ FSLVBM/UserGuide). Image outputs were viewed by using fslview (http://fsl.fmrib.ox.ac.uk/fsl/fslview/).

The optic chiasm area was measured by using the ViewForum (Philips Healthcare) region-of-interest software. Manual regions outlining the optic chiasm were added to coronal sections and were quantitated by using methods described previously. $^{27}$ Interobserver repeatability of this method in our center by using a random sample of 20 subjects showed excellent agreement between 2 observers (linear regression $r^{2}=$ 0.97, $P<.0001)$.

\section{Statistical Analysis}

All between-group analyses were completed by using the unpaired $t$ test or Mann-Whitney $U$ test when appropriate (Statistical Package for the Social Sciences, Version 20; IBM UK, Woking, United Kingdom). The results were considered significant at $P<$ .05 . Voxel-based morphometry analyses were completed by using the unpaired $t$ test by using the Randomize software of FSL within to an archiving failure. the framework of the General Linear Model. Because this was an exploratory study, results were considered significant at $P<.05$ corrected for the number of comparisons, as described previously. ${ }^{28-30}$

\section{RESULTS}

Twenty-nine of the 35 recruited patients with MoMHR and 29 of the 35 recruited patients with conventional THA underwent brain MR imaging and neurologic assessment and are included in these

analyses. A key industry in our local population is metal grinding, and 5 subjects with a history of metal intraocular foreign bodies that precluded brain MR imaging were excluded from the study, 3 subjects were unable to complete the scanning protocol due to claustrophobia, and MR imaging data were lost in 4 subjects due

The patient groups were well-matched for age at surgery, sex, and time since surgery (Table $1, P>.05$, all comparisons). Clinical neurologic examination demonstrated no abnormal clinical findings, with the exception of a benign essential tremor in 1 male subject with MoMHR and reduced facial expression, bradykinesia, and mild spasticity consistent with a mild extrapyramidal disorder in 1 man in the conventional THA group. UPDRS scores for dysfunction in Part 2 (activities of daily living) and Part 3 (motor symptoms) were similar between the 2 groups $(P>.05$, both comparisons). Median visual acuity at 6 meters in the MoMHR group was 12 (interquartile range, 6.8-51) in the left eye and 12 (interquartile range, 6.8-51) in the right eye; and in the THA group, it was 9 (interquartile range, 9-33) in the left eye and 12 (interquartile range, 9-33) in the right eye $(P>.05$, both comparisons). Median blood and plasma Co and Cr levels were 5- to 50 -fold higher in patients with MoMHR versus those with THA $(P<.0001$, all comparisons, Table 1$)$.

Brain gray matter attenuation measured by voxel-based morphometry was lower in the occipital cortex of the patients with MoMHR versus those with THA, corresponding to the primary visual cortex (Fig $1 ; P<.005$, uncorrected; $P>.05$, corrected). In view of the location of this difference in gray matter attenuation, patients were invited to return for clinical ophthalmologic examination to exclude primary eye pathology as a confounding variable for this analysis. Forty-eight male patients returned for this examination. Fourteen of these subjects were identified as having a primary ocular pathology that might contribute to visual cortical dysfunction or optic atrophy, leaving 34 subjects (17 in each group) for the subgroup analysis. Gray matter attenuation remained reduced in the occipital region (Fig 2 , top panel; $P<.005$, uncorrected; $P>.05$, corrected) but was also reduced in the right and left putamen and the left head of the caudate nucleus (Fig 2, bottom panel; $P<.005$, uncorrected; all regions, $P>.05$, corrected). The optic chiasm area, measured by using Viewforum, was $36.7 \pm 4.7 \mathrm{~mm}^{2}$ and $39.5 \pm 6.7 \mathrm{~mm}^{2}$ in the patients with MoMHR and THA, respectively (mean difference $=-2.7 \mathrm{~mm}$; 95\% CI, $-5.7-$ $0.2 \mathrm{~mm} ; P=.076$ ). After we removed patients with primary ocular pathologies and those who did not return for ophthalmic assessment, 

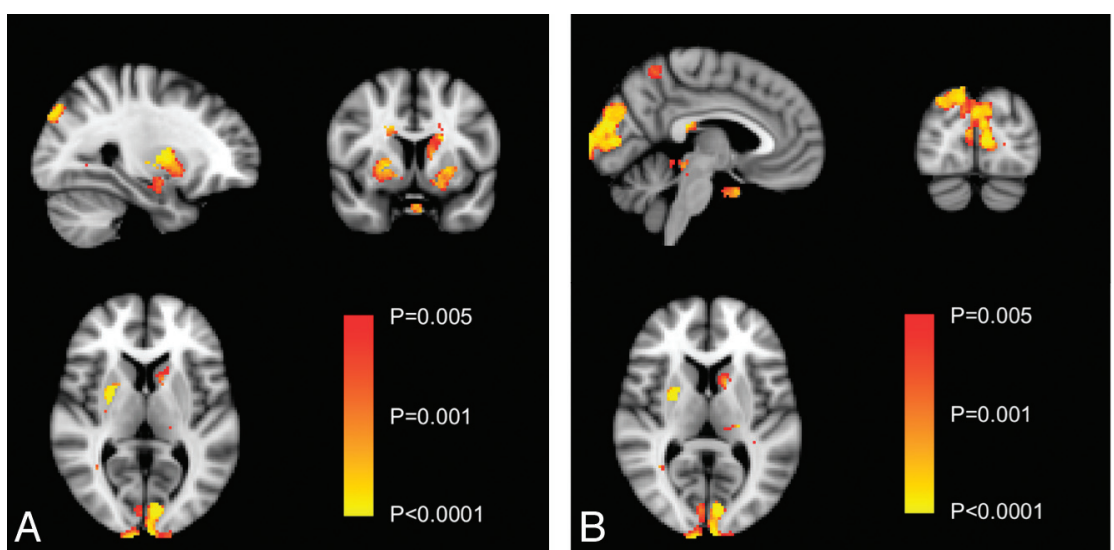

FIG 2. Voxel-based morphometric analysis of differences in brain gray matter attenuation between the MoMHR and THA groups, after exclusion of patients with primary ocular pathology. The top panel highlights occipital cortex differences between groups; the bottom panel highlights differences in basal ganglia between groups. Comparative areas of gray matter loss in the MoMHR group are highlighted by using the color bar across the significance value range $P<.05$ to $P<.001$ (uncorrected).

Table 2: Brain volumes ${ }^{\mathrm{a}}$

\begin{tabular}{lccc}
\hline \multicolumn{1}{c}{ Structure $\left(\mathbf{m m}^{3}\right)$} & MoMHR & THA & $P$ Value \\
\hline Total brain volume & $1.20 \times 10^{6} \pm 75219$ & $1.18 \times 10^{6} \pm 96956$ & .08 \\
Gray matter & $5.89 \times 10^{5} \pm 57984$ & $5.74 \times 10^{5} \pm 67886$ & .11 \\
White matter & $6.14 \times 10^{5} \pm 25380$ & $6.01 \times 10^{5} \pm 34845$ & .09 \\
Brain stem & $22798 \pm 2407$ & $22187 \pm 2380$ & .34 \\
Thalamus & $14960 \pm 989$ & $14710 \pm 1086$ & .36 \\
Caudate nucleus & $6544 \pm 505$ & $6615 \pm 601$ & .63 \\
Putamen & $8512 \pm 486$ & $8384 \pm 624$ & .39 \\
Pallidum & $3498 \pm 339$ & $3518 \pm 424$ & .84 \\
Hippocampus & $7026 \pm 670$ & $6952 \pm 793$ & .70 \\
Amygdala & $2505 \pm 289$ & $2342 \pm 402$ & .08 \\
Nucleus accumbens & $729 \pm 166$ & $715 \pm 163$ & .76 \\
\hline
\end{tabular}

${ }^{a}$ Data are mean \pm SD and are adjusted for intracranial volume. Analyses are MoMHR versus THA by unpaired $t$ test or the Mann-Whitney $U$ test.

PRIME R2'

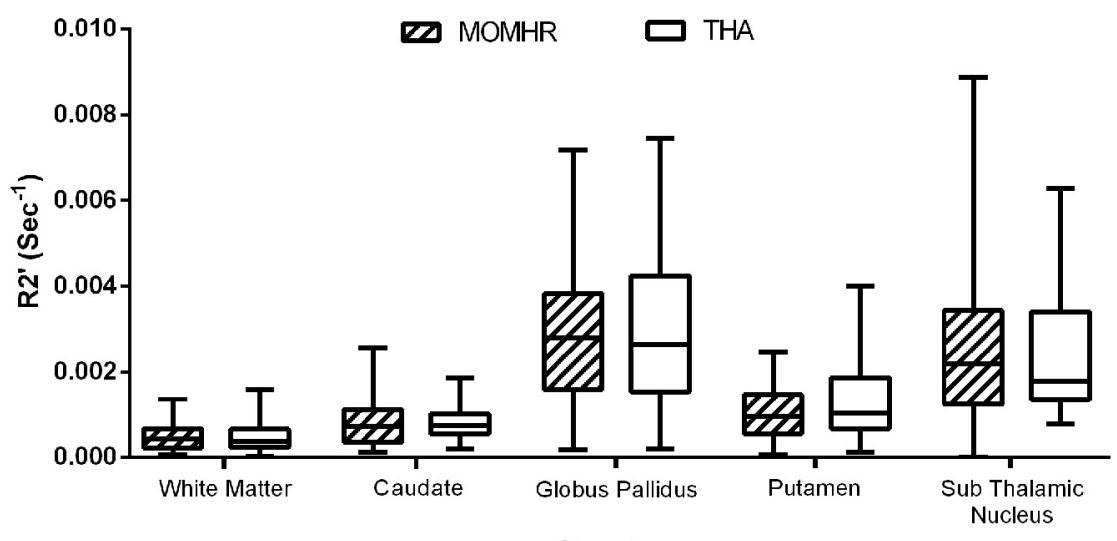

Structure

FIG 3. Quantitation of brain metal deposition by PRIME R2' values (second ${ }^{-1}$ ). Analyses are MoMHR versus THA by the Wilcoxon test, $P>.05$ for all comparisons.

the optic chiasm area was $36.3 \pm 4.7 \mathrm{~mm}^{2}$ in the MoMHR group and $40.3 \pm 6.3 \mathrm{~mm}^{2}$ in the THA group $(n=17$ per group; unpaired mean difference, $-3.9 \mathrm{~mm}^{2}$; $95 \% \mathrm{CI},-7.84$ to $\left.-0.04 ; P=.048\right)$.

Total brain volume and white and gray matter volumes were similar in both patient groups (as measured by FAST, FSL), as were subcortical nuclei, basal ganglia, and brain stem volumes computed by using FIRST, FSL (Table 2, values corrected for intracranial volume; $P>.05$, all comparisons). R2' relaxation times in the caudate nucleus, subthalamic nucleus, pallidum, putamen, and white matter were also similar between groups; this finding indicates that any possible difference in the level of metal deposition between the subjects with MoMHR and those with THA was below the level of detection available by using the PRIME sequence (Fig 3; $P>$ .05 , all comparisons).

\section{DISCUSSION}

In this study, we found that healthy asymptomatic patients with confirmed chronic exposure to elevated circulating concentrations of $\mathrm{Co}$ and $\mathrm{Cr}$ after MoMHR had differences in brain structure compared with a group of age-, sex-, and time since surgery-matched patients with the same underlying disease, but who had received a conventional hip prosthesis and who had circulating metal concentrations that are similar to normal physiologic levels. Patients who had received MoMHR tended toward lower gray matter attenuation in the occipital cortex and basal ganglia and had a smaller optic chiasm area than those patients who had received a conventional prosthesis, after exclusion of patients with primary ocular pathologies from the analysis. We found no evidence of association between MoMHR exposure and changes in brain structural volumes or with metal accumulation within the subcortical nuclei or basal ganglia. We also found no differences in neurologic function, measured by clinical examination and UPDRS, and no differences in visual function, measured by ophthalmologic assessment, between the groups.

These structural trends, though small, are consistent with known toxicologic effects of $\mathrm{Co}$ and $\mathrm{Cr}$ on the visual system observed in patients with acute exposure to high metal concentrations associated with prosthesis malfunction. ${ }^{8-10}$ Our data suggest that moderately elevated circulating metal concentrations for 8 years after MoMHR are associated with imaging features in keeping with possible cell loss in the visual system. A chronic insult to the anterior visual pathways has been previously reported in humans to cause voxel-based changes in the primary visual cortex in the context of glaucoma. ${ }^{31}$ The rational underpinning of these findings is 
that such visual cortex changes are produced by transneuronal degeneration in patients with optic nerve damage. Visual cortex abnormalities on voxel-based morphometry consistent with cortical atrophy have also been found in patients with macular degeneration. ${ }^{32}$ A recent animal study by Apostoli et al, ${ }^{33}$ has shown rabbits with prolonged systemic exposure to $\mathrm{Co}$ or $\mathrm{Cr}$ in isolation or combined have optic and auditory neuropathy. Cobalt produces toxicity in rats, leading to glioma, ${ }^{34}$ neuroblastoma, ${ }^{35}$ and neuronal cell damage. ${ }^{36}$ In addition, cobalt has a dose-dependent effect on apoptosis and secondary necrosis in astrocytes, through the generation of reactive oxygen species and subsequent $\alpha$ subunit of hypoxia inducible factor-1 pathway activation. ${ }^{6}$

Our FIRST data suggest that this concentration of metal exposure is not associated with a detectable loss in volume of the major brain structures. Both of our patient groups had brain and subcortical volumes similar to those found in the general population for individuals of similar age and sex. ${ }^{37}$ Similarly, although both Co and Cr may be deposited in the basal ganglia, especially in the pallidum, putamen, and caudate nucleus, ${ }^{3,4,38}$ our PRIME analysis of R2' data suggest that this exposure does not result in an increase in detectable deposition of paramagnetic ions within these structures. Our methods have been used previously to assess iron concentration within the basal ganglia of patients with HIV and Parkinson disease. Concentrations of iron up to $200 \mu \mathrm{\gamma} / \mathrm{g}$ tissue wet weight are found in the globus pallidus, red nucleus, substantia nigra, and putamen regions. ${ }^{39}$ A previous study showed a sensitivity of the PRIME sequence R2' relaxation time to iron deposition of approximately $0.02 \mathrm{~s} / \mu \mathrm{gFe} / \mathrm{g}$ wet weight of tissue. ${ }^{19}$ In a study to measure $\mathrm{R}^{*}$ in intact postmortem brains followed by quantitative postmortem brain measurement with inductively coupled plasma mass spectrometry, Langkammer et $\mathrm{al}^{40}$ found a formula for R2* $\left(\right.$ second $\left.^{-1}\right)=14.3+0.27 \times$ [iron concentration] ( $\mathrm{mg} \mathrm{Kg}^{-1}$ wet mass). The use of this approach to detect other metals such as Co and Cr is not so well-developed. Co, like iron, displays ferromagnetism, while $\mathrm{Cr}$ has a lower paramagnetic susceptibility of $+167 \chi \mathrm{m} / 10^{-6} \mathrm{~cm}^{3} \mathrm{~mol}^{-1}$. As a sulfate, this increases to $+11,800 \chi \mathrm{m} / 10^{-6} \mathrm{~cm}^{3} \mathrm{~mol}^{-1}$. No differences were observed for R2' between the patient groups we studied, but this may reflect the expected lower sensitivity of the PRIME sequence to effects from Co and Cr compared with iron and the relatively low concentrations of $\mathrm{Co}$ and $\mathrm{Cr}$ in the brain. Typical normal levels in the cortex are $2 \mu \mathrm{g} / \mathrm{Kg}$ for Co and $10 \mu \mathrm{g} / \mathrm{Kg}$ for $\mathrm{Cr},{ }^{4}$ which are several orders of magnitude lower than those of iron $(50-250 \mathrm{mg} / \mathrm{Kg})$. If the serum concentration of these elements was raised by a factor of 50 and they crossed the bloodbrain barrier, the maximum levels of $\mathrm{Co}$ and $\mathrm{Cr}$ in the brain would be $\sim 500 \mu \mathrm{g} / \mathrm{Kg}$, and thus our failure to detect these metals may be due to insensitivity of the PRIME sequence method to these relatively low metal concentrations compared with iron in brain tissue.

This is the first study to explore whether chronic exposure to moderate circulating metal is associated with brain structural and functional change. It was, by its nature, exploratory and not powered for analysis of specific brain areas or with a priori knowledge of potential effect sizes. While we observed some differences in brain morphometry between the groups, these did not remain after multiple-comparison correction, possibly related to sample size. Further study focusing analysis on the visual cortex and basal ganglia is warranted to confirm these preliminary findings in patients prescreened for potential confounders such as coexistence of primary ocular pathology.

\section{CONCLUSIONS}

This exploratory study was conducted in a clinically asymptomatic population and was cross-sectional in design. The patients with MoMHR who we studied had circulating metal concentrations that, though elevated, were not indicative of a failing prosthesis. We thus aimed to detect subclinical signals to indicate whether metal exposure due to prosthesis choice is associated with structural or functional changes in the brain and, if so, which systems show the greatest signal change. Our detection of subtle structural changes associated with metal exposure suggests that further examination of the relationship between metal exposure, brain structure, and clinical symptoms in a larger group of patients across a higher concentration range of metal exposure and for longer periods is warranted to definitively address the relationship between exposure and structural change and to determine their clinical relevance.

Disclosures: Jeremy Mark Wilkinson—RELATED: Grant: National Institutes of Health Research, United Kingdom, ${ }^{*}$ Comments: This work was funded through a Biomedical Research Unit core grant from the National Institute for Health Research. *Money paid to the institution.

\section{REFERENCES}

1. Heisel C, Streich N, Krachler M, et al. Characterization of the running-in period in total hip resurfacing arthroplasty: an in vivo and in vitro metal ion analysis. J Bone Joint Surg Am 2008;90 (suppl 3):125-33

2. Daniel J, Ziaee H, Pradhan C, et al. Blood and urine metal ion levels in young and active patients after Birmingham hip resurfacing arthroplasty: four-year results of a prospective longitudinal study. J Bone Joint Surg Br 2007;89:169-73

3. Duckett $\mathrm{S}$. Abnormal deposits of chromium in the pathological human brain. J Neurol Neurosurg Psychiatry 1986;49:296-301

4. Höck A, Demmel U, Schicha H, et al. Trace element concentration in human brain: activation analysis of cobalt, iron, rubidium, selenium, zinc, chromium, silver, cesium, antimony and scandium. Brain 1975;98:49-64

5. Quinteros FA, Poliandri AH, Machiavelli LI, et al. In vivo and in vitro effects of chromium VI on anterior pituitary hormone release and cell viability. Toxicol Appl Pharmacol 2007;218:79-87

6. Karovic O, Tonazzini I, Rebola N, et al. Toxic effects of cobalt in primary cultures of mouse astrocytes: similarities with hypoxia and role of HIF-1alpha. Biochem Pharmacol 2007;73:694-708

7. Huk OL, Catelas I, Mwale F, et al. Induction of apoptosis and necrosis by metal ions in vitro. J Arthroplasty 2004;19:84-87

8. Oldenburg M, Wegner R, Baur X. Severe cobalt intoxication due to prosthesis wear in repeated total hip arthroplasty. $J$ Arthroplasty 2009;24:825 e815-20

9. Rizzetti MC, Liberini P, Zarattini G, et al. Loss of sight and sound: could it be the hip? Lancet 2009;373:1052

10. Steens W, von Foerster G, Katzer A. Severe cobalt poisoning with loss of sight after ceramic-metal pairing in a hip: a case report. Acta Orthop 2006;77:830-32

11. Rising JP, Reynolds IS, Sedrakyan A. Delays and difficulties in assessing metal-on-metal hip implants. N Engl J Med 2012;367:e1

12. Hartmann A, Lutzner J, Kirschner S, et al. Do survival rate and serum ion concentrations 10 years after metal-on-metal hip resurfacing provide evidence for continued use? Clin Orthop 2012;470:3118-26

13. Holland JP, Langton DJ, Hashmi M. Ten-year clinical, radiological 
and metal ion analysis of the Birmingham hip resurfacing: from a single, non-designer surgeon. J Bone Joint Surg Br 2012;94:471-76

14. Gwilym SE, Filippini N, Douaud G, et al. Thalamic atrophy associated with painful osteoarthritis of the hip is reversible after arthroplasty: a longitudinal voxel-based morphometric study. Arthritis Rheum 2010;62:2930-40

15. Prentice JR, Clark MJ, Hoggard N, et al. Metal-on-metal hip prostheses and systemic health: a cross-sectional association study 8 years after implantation. PloS One 2013;8:e66186

16. Bowler RM, Roels HA, Nakagawa S, et al. Dose-effect relationships between manganese exposure and neurological, neuropsychological and pulmonary function in confined space bridge welders. $O c$ cup Environ Med 2007;64:167-77

17. Lucchini RG, Albini E, Benedetti L, et al. High prevalence of Parkinsonian disorders associated to manganese exposure in the vicinities of ferroalloy industries. Am J Ind Med 2007;50:788 - 800

18. Patenaude B, Smith SM, Kennedy DN, et al. A Bayesian model of shape and appearance for subcortical brain segmentation. Neuroimage 2011;56:907-22

19. Graham JM, Paley MN, Grunewald RA, et al. Brain iron deposition in Parkinson's disease imaged using the PRIME magnetic resonance sequence. Brain 2000;123(pt 12):2423-31

20. Miszkiel KA, Paley MN, Wilkinson ID, et al. The measurement of $\mathrm{R} 2, \mathrm{R2}^{\star}$ and $\mathrm{R}^{\prime}$ ' in HIV-infected patients using the prime sequence as a measure of brain iron deposition. Magn Reson Imaging 1997;15:1113-19

21. Wallis LI, Paley MN, Graham JM, et al. MRI assessment of basal ganglia iron deposition in Parkinson's disease. Magn Reson Imaging 2008;28:1061-67

22. Ueno S, Susa N, Furukawa Y, et al. Formation of paramagnetic chromium in liver of mice treated with dichromate (VI). Toxicol Appl Pharmacol 1995; 135:165-71

23. Good CD, Johnsrude IS, Ashburner J, et al. A voxel-based morphometric study of ageing in $\mathbf{4 6 5}$ normal adult human brains. Neuroimage 2001;14:21-36

24. Smith SM, Jenkinson M, Woolrich MW, et al. Advances in functional and structural MR image analysis and implementation as FSL. Neuroimage 2004;23(suppl 1):S208-19

25. Smith SM. Fast robust automated brain extraction. Hum Brain Mapp 2002;17:143-55

26. Zhang Y, Brady M, Smith S. Segmentation of brain MR images through a hidden Markov random field model and the expectationmaximization algorithm. IEEE Trans Med Imaging 2001;20:45-57

27. Wagner AL, Murtagh FR, Hazlett KS, et al. Measurement of the nor- mal optic chiasm on coronal MR images. AJNR Am J Neuroradiol 1997; 18:723-26

28. Henley SM, Ridgway GR, Scahill RI, et al. Pitfalls in the use of voxelbased morphometry as a biomarker: examples from Huntington disease. AJNR Am J Neuroradiol 2010;31:711-19

29. Radua J, Mataix-Cols D, Phillips ML, et al. A new meta-analytic method for neuroimaging studies that combines reported peak coordinates and statistical parametric maps. Eur Psychiatry 2012;27:605-11

30. Genovese CR, Lazar NA, Nichols T. Thresholding of statistical maps in functional neuroimaging using the false discovery rate. Neuroimage 2002;15:870-78

31. Zikou AK, Kitsos G, Tzarouchi LC, et al. Voxel-based morphometry and diffusion tensor imaging of the optic pathway in primary openangle glaucoma: a preliminary study. AJNR Am J Neuroradiol 2012;33:128-34

32. Boucard CC, Hernowo AT, Maguire RP, et al. Changes in cortical grey matter density associated with long-standing retinal visual field defects. Brain 2009;132:1898-906

33. Apostoli P, Catalani S, Zaghini A, et al. High doses of cobalt induce optic and auditory neuropathy. Exp Toxicol Pathol 2013;65:719-27

34. Yang SJ, Pyen J, Lee I, et al. Cobalt chloride-induced apoptosis and extracellular signal-regulated protein kinase $1 / 2$ activation in rat C6 glioma cells. J Biochem Mol Biol 2004;37:480-86

35. Olivieri G, Hess C, Savaskan E, et al. Melatonin protects SHSY5Y neuroblastoma cells from cobalt-induced oxidative stress, neurotoxicity and increased beta-amyloid secretion. J Pineal Res 2001;31:320-25

36. Wang G, Hazra TK, Mitra S, et al. Mitochondrial DNA damage and a hypoxic response are induced by $\mathrm{CoCl}(2)$ in rat neuronal $\mathrm{PC12}$ cells. Nucleic Acids Res 2000;28:2135-40

37. de Jong LW, van der Hiele K, Veer IM, et al. Strongly reduced volumes of putamen and thalamus in Alzheimer's disease: an MRI study. Brain 2008;131:3277-85

38. Gooddy W, Hamilton EI, Williams TR. Spark-source mass spectrometry in the investigation of neurological disease. II. Element levels in brain, cerebrospinal fluid and blood: some observations on their abundance and significance. Brain 1975;98:65-70

39. Haacke EM, Cheng NY, House MJ, et al. Imaging iron stores in the brain using magnetic resonance imaging. Magn Reson Imaging 2005;23:1-25

40. Langkammer C, Krebs N, Goessler W, et al. Quantitative MR imaging of brain iron: a postmortem validation study. Radiology 2010;257:455-62 